\title{
Characterizing electrode reactions by multisampling the current in square-wave voltammetry
}

\author{
Valentin Mirceski ${ }^{a, d, *}$, Dariusz Guziejewski ${ }^{\mathrm{b}}$, Monika Bozem ${ }^{\mathrm{c}}$, Ivan Bogeski ${ }^{\mathrm{c}}$ \\ a Institute of Chemistry, Faculty of Natural Sciences and Mathematics, “Ss Cyril and Methodius" University, P.O. Box 162, 1000 Skopje, Macedonia \\ ${ }^{\mathrm{b}}$ Department of Inorganic and Analytical Chemistry, University of Lodz, Tamka 12, 91-403 Lodz, Poland \\ ${ }^{\mathrm{c}}$ Department of Biophysics, School of Medicine, Saarland University, 66421 Homburg, Germany \\ d Faculty of Medical Sciences, “Goce Delcev" University, Krste Misirkov bb, 2000 Stip, Macedonia
}

\section{A R T I C L E I N F O}

Article history:

Received 4 April 2016

Received in revised form 22 July 2016

Accepted 22 July 2016

Available online 25 July 2016

\section{Keywords:}

square-wave voltammetry

electrode kinetics

chronoamperometry

simulations

\section{A B S T R A C T}

A novel and simple methodology for characterization of electrode processes by square-wave voltammetry (SWV), considered as a complex repetitive double-step chronoamperometric experiment, is proposed. Specifically, the sampling of the current at the very end of each potential pulse, as common in conventional SWV, is replaced with a multisampling protocol during each potential pulse, resulting in generation of a series of corresponding multisampled SW voltammograms constructed out of a single measurement. The evolution of such voltammograms reflects the chronoamperometric properties of the current in the course of each potential pulse, thus being specific to electrode mechanism and electrode kinetics. The proposed methodology could serve as a basis for modification of common SWV mode in commercially available instrumentation for the purpose of an advanced characterization of electrode processes. The methodology is theoretically illustrated by the analysis of three kinetically controlled common electrode mechanisms, such as the electrode reaction of a solution resident and surface confined redox couples, as well as the regenerative catalytic electrode mechanism (EC'), while the experimental verification is done with the electrode reaction of $\mathrm{Eu}^{3+} / \mathrm{Eu}^{2+}$ and azobenzene/ hydrazobenzene redox couples.

(c) 2016 Elsevier Ltd. All rights reserved.

\section{Introduction}

Voltammetric methods [1] for kinetic and mechanistic characterisation of electrode processes at a constant scan rate of the voltammetric experiment are particularly appealing due to their elegance and simplicity [2,3]. Such methods provide an alternative route for studying electrode reactions, taking the advantage of the unique feature of electrode kinetics to be highly sensitive to the electrode potential. While this approach is not possible with classical cyclic voltammetry [4], where the electrode kinetics could be accessed only by changing the time frame of the voltammetric experiment (i.e., the scan rate), the pulse voltammetric techniques provide a means to measure the electrode kinetics by altering the height of applied potential pulses at a constant time window of the experiment. In the recent years we have made an attempt to develop such methods under conditions of square-wave voltammetry (SWV) [5], which was achieved by the virtue of the SW

\footnotetext{
* Corresponding author.

E-mail address: valentin@pmf.ukim.mk (V. Mirceski).
}

amplitude, inspecting the influence of the amplitude variation to the current [2] or position [3] of the voltammetric response. In a broader context let us note that the latter methods are only a small fraction of a plethora of novel studies of merit dedicated to the methodological and theoretical development of SWV [6-19].

In the present work we propose an alternative approach in processing voltammetric data, aiming to develop simple and fast strategy for characterization of electrode processes. The core idea is based on inspecting electrochemical data collected by conducting a SW voltammetric experiment combined with a multisampling current procedure [20]. Indeed, a similar idea for advanced analysis of electrochemical data collected by a voltammetric experiment was already introduced by Bond et al. based on the advanced large amplitude Fourier transformed a.c. voltammetry [21].

For the present system, let us first recall that the excitation signal in SWV consists of a staircase potential mofulation combined with potential pulses (Fig. 1A). Two oppositely oriented potential pulses, equal in height and duration $\left(t_{\mathrm{p}}\right)$, are imposed to each potential stair, forming one potential cycle in SWV (Fig. 1B). As well known, aiming to discriminate against the charging current, 
the sampling is done at the very end of each potential pulse, as typical for pulse voltammetric techniques (Fig. 1B). However, let us consider a complex, repetitive double-step chronoamperometric experiment with a potential modulation identical as in SWV, which unlike SWV, provides electrochemical information at each time of the experiment. A typical outcome of such experiment, presented in a current-time domain, is depicted in Fig. 1C, revealing the wealth of electrochemical data collected in the course of a single experiment. Applying a multisampling current procedure at different sampling times $\left(t_{\mathrm{s}}\right)$, as depicted in Fig. 1D, several, so called multisampled SW voltammograms can be constructed out of a single experiment. It can be reasonably assumed that the features of such multisampled SW voltammograms have to depend on the duration of the pulse $t_{\mathrm{p}}$ (i.e., SW frequency $f=1 / 2 t_{\mathrm{p}}$ ) as well as on the sampling time $t_{\mathrm{s}}$, as already implied in the early seminal work of Rameley and Crause [22]. The latter is illustrated in Fig. 1E, comparing the voltammetric response obtained by repetitive double-step chronoamperometric experiment with $t_{\mathrm{p}}=50 \mathrm{~ms}(f=10 \mathrm{~Hz})$ and sampling time $t_{\mathrm{s}}=40 \mathrm{~ms}$, with the response of the conventional SWV conducted with $t_{\mathrm{p}}=t_{\mathrm{s}}=40$ $\mathrm{ms}(f=12.5 \mathrm{~Hz})$. The two responses are similar, but not identical, due to differences in the thickness of the diffusion layer developed in the course of the experiment and the differences in the overall scan rate [5].

For further illustration, let us assume an experiment at a low frequency, $f=10 \mathrm{~Hz}$ (the duration of a single potential pulse, $t_{\mathrm{p}}=50 \mathrm{~ms}$ ) and a scan increment, $\Delta E=2 \mathrm{mV}$, which means the overall scan rate of the potential modulation is relatively low, i.e., $20 \mathrm{mV} / \mathrm{s} \quad(\nu=\Delta E f)$. Considering the fact that under common experimental conditions the charging current drops to negligible values after few ms time interval, it follows that most of the currents measured in the course of the potential pulse with $t_{\mathrm{p}}=50 \mathrm{~ms}$ can provide useful information on the faradaic electrode process, which justifies the idea of multisampling current procedure. Moreover, the charging current can be effectively discriminated with much shorter potential pulses (e.g. $t_{\mathrm{p}}=2.5 \mathrm{~ms}$; $f=200 \mathrm{~Hz}$ ) as well [5], provided the multisampling current procedure is limited to the last quarter of the potential pulse.

Hence, the main question in the present study is whether the features of SW voltammograms obtained by multisampling procedure of a single experiment are informative regarding kinetics and mechanism of the ongoing electrode reaction? The present, mainly theoretically oriented study, aims to address the latter question, considering several common electrode reactions such as simple electrode reaction at a planar electrode of two chemically stable solution resident redox species, electrode mechanism coupled with a homogeneous regenerative catalytic chemical reaction (EC' mechanism) $[23,24]$, as well as the electrode reaction of a surface confined redox couple [25]. A part of the theoretical data are illustrated with experiments conducted with europium(III) [26-28] and azobenzene [29] at a hanging mercury drop electrode (HMDE).

\section{Experimental}

All experiments have been performed using multi-Autolab potentiostat model M101 and Nova (v. 1.10.3, both Metrohm Autolab B.V.) software-controlled electrochemical measurement system. The working electrode was HMDE ( $\mathrm{mtm}$ anko instruments, Poland, surface area $0.00102 \mathrm{~cm}^{2}$ ) combined with $\mathrm{Ag} / \mathrm{AgCl}$ (3 mol $\mathrm{L}^{-1} \mathrm{KCl}$ ) as a reference and a platinum wire as a counter electrode in a conventional three-electrode setup.

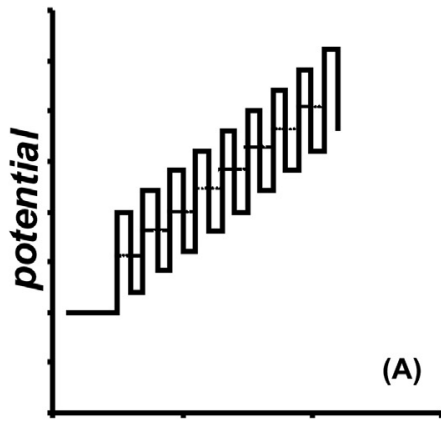

time

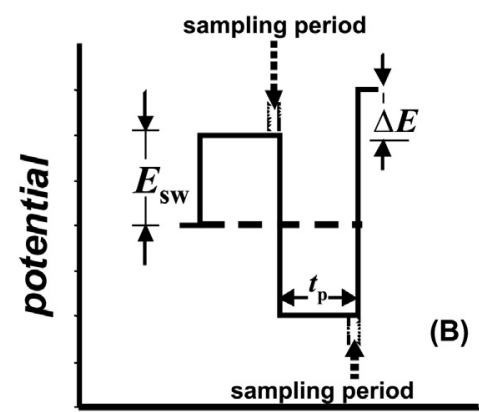

time

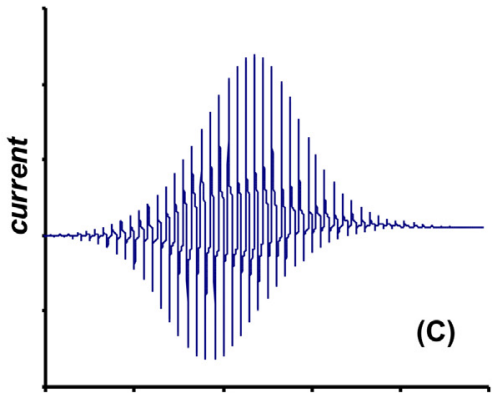

time
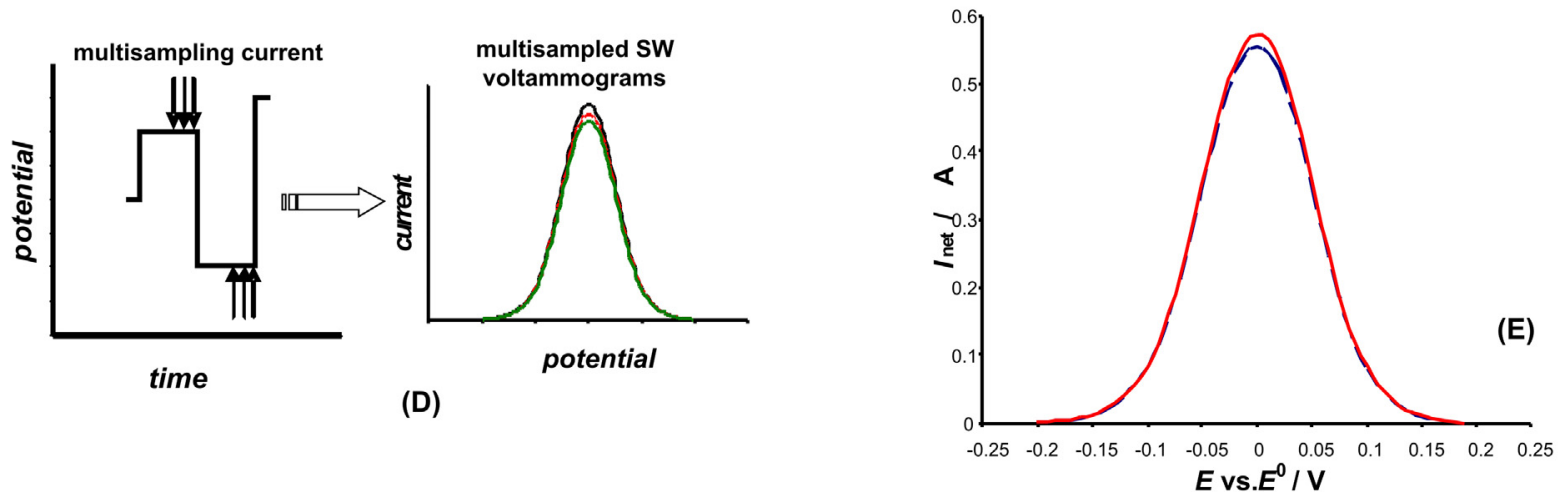

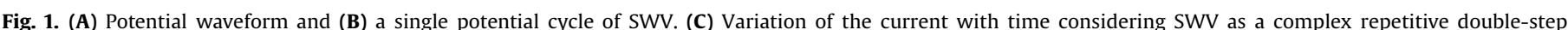

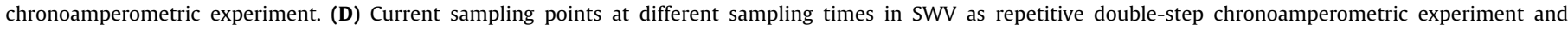

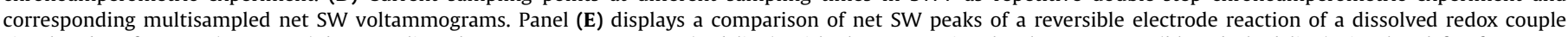

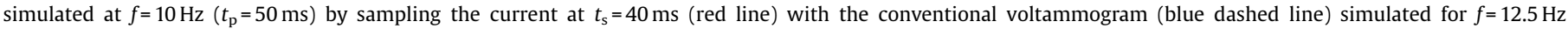

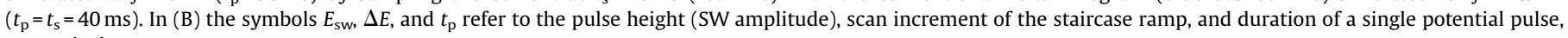
respectively. 
All chemicals used were of analytical grade purity (Merck, Sigma-Aldrich or POCh). All solutions were prepared with water from Merck-Millipore Direct Q-3 purification system. $1 \mathrm{mmol} \mathrm{L}^{-1}$ azobenzene stock solution was prepared by dissolving in methanol, whereas $10 \mathrm{mmol} \mathrm{L}^{-1}$ europium(III) chloride solution was prepared in water. Phosphate buffer at $\mathrm{pH}=4.8$ was used as a supporting electrolyte in the experiments with azobenzene, whereas a mixture of $5 \mathrm{mmol} \mathrm{L}^{-1} \mathrm{HClO}_{4}$ and $1 \mathrm{~mol} \mathrm{~L}^{-1} \mathrm{NaBr}$ was utilized in experiments with europium [27].

The general procedure for measurements was as follows: $10 \mathrm{~mL}$ of the supporting electrolyte was placed in the voltammetric cell and the solution was purged with argon for $300 \mathrm{~s}$ with stirring the solution. Accumulation of the compound (referring to azobenzene only), followed by a 10 s quiescent period, was performed. As the multisampling current procedure is not allowed in conventional SWV mode of the measuring station used, experiments have been conducted by designing a series of subsequent double-step chronoamperometric measurements in Nova software at a single mercury drop in a way corresponding to a SW potential modulation with the frequency of $f=8 \mathrm{~Hz}\left(t_{\mathrm{p}}=62.5 \mathrm{~ms}\right)$, amplitude $E_{\mathrm{sw}}=50 \mathrm{mV}$, and scan increment $\Delta E=5 \mathrm{mV}$. Thus, the designed repetitive chronoamperometric measurements enabled to sample the current at any time of the experiment. In order to control the correctness of the designed procedure, a conventional SW voltammogram attributed with the same parameters was performed immediately after the chronoamperometric measurements.

In the case of conventional SWV kinetic analysis, a series of voltammograms at different amplitudes were measured following the methodology described in [2,3].

\section{Results and discussion}

\subsection{Theoretical data}

We start our consideration with an idealized reversible oneelectron electrode reaction of a dissolved (sol) redox couple at a planar electrode (eq. (1); the charge of the species is omitted).

$\mathbf{R}(\mathrm{sol}) \rightleftarrows \mathbf{O}(\mathrm{sol})+\mathrm{e}$

It has been assumed that at the beginning of the experiment only $\mathbf{R}$ form of the redox couple $\mathbf{R} / \mathbf{O}$ is present in the solution at a bulk concentration $c^{*}$, while both redox forms have a common diffusion coefficient $D$. The proposed methodology will be exemplified first considering a long duration potential pulses of $t_{\mathrm{p}}=50 \mathrm{~ms}$ (i.e. $f=10 \mathrm{~Hz}$ ), conducting numerical simulations by means of the recurrent formula given in [5]. Each potential pulse is divided into 50 time increments; thus, for instance, the time increment with a serial number 40 corresponds to the sampling time $t_{\mathrm{s}}=40 \mathrm{~ms}$. A single simulation provides a collection of current values corresponding to each time increment, covering the whole time window of the SW potential modulation. Though, as previously mentioned, the sampling could be done much earlier, the interest is focused on the last quarter of the potential pulses. By sampling the current at $40^{\text {th }}, 41^{\text {st }}, 42^{\text {nd }}$ up to the $50^{\text {th }}$ time increment eleven SW voltammograms have been constructed. Note that the voltammogram sampled at $50^{\text {th }}$ time increment corresponds to the conventional SW voltammogram [5]. Besides, let us note the number of time increments used in the recurrent formula for simulation of the response [5] can be deliberately increased for the purpose of higher precision, without any difficulties in the simulation procedure on the expense of the simulation time only. Though the simulations with 50 time increments provide sufficient precision in calculating dimensionless current, the simulations with 1000 time increments can be conducted easily, and the average relative difference between the two set of data is only $1.28 \% \pm 0.84$, considering the conventional net SW voltammogram of a reversible electrode reaction.

The evolution of multisampled net SW voltammograms is depicted in Fig. 2A, revealing a decrease of the net peak current with sampling time, which is a consequence of the expansion of the diffusion layer in the course of the potential pulse. The shape, the half-peak width and the peak potentials are apparently independent on the sampling time, together with the shape of the forward and backward voltammetric components, as depicted in the inset of the panel 2A. The degree of the net peak current $\left(\Delta I_{\mathrm{p}}\right)$ decreasing with the sampling time depends on the amperometric constant defined by the product $n F A c^{*}(D)^{0.5}$, where $n$ is the number of electrons, $A$ is the electrode surface area, $F$ is Faraday constant and $D$ is diffusion coefficient. The panel $2 \mathrm{~B}$ shows the role of the amperometric constant, which has been altered by changing the diffusion coefficient value. As expected, for larger $D$, the decline of the net peak current is steeper, as a consequence of faster diffusion and expansion of the diffusion layer in the course of the single potential pulse. Obviously, from the slope of such dependence the value of the $D$ could be estimated, provided the other constants are known. If the same data of Fig. 2B are replotted in a logarithmic form $\left(\log \left(\Delta I_{\mathrm{p}}\right)\right.$ vs. $\left.\log \left(t_{\mathrm{s}}\right)\right)$ straight lines are obtained with an equal slope of -0.6522 , regardless of the amperometric constant value, which could be considered as a quantitative criterion for the reversible electrode reaction.

These results can be rationalized taking into account the general definition of the current in SWV, i.e., $I=n F A c^{*}(D f)^{0.5} \Psi$, where $\Psi$ is the dimensionless voltammetric function reflecting the
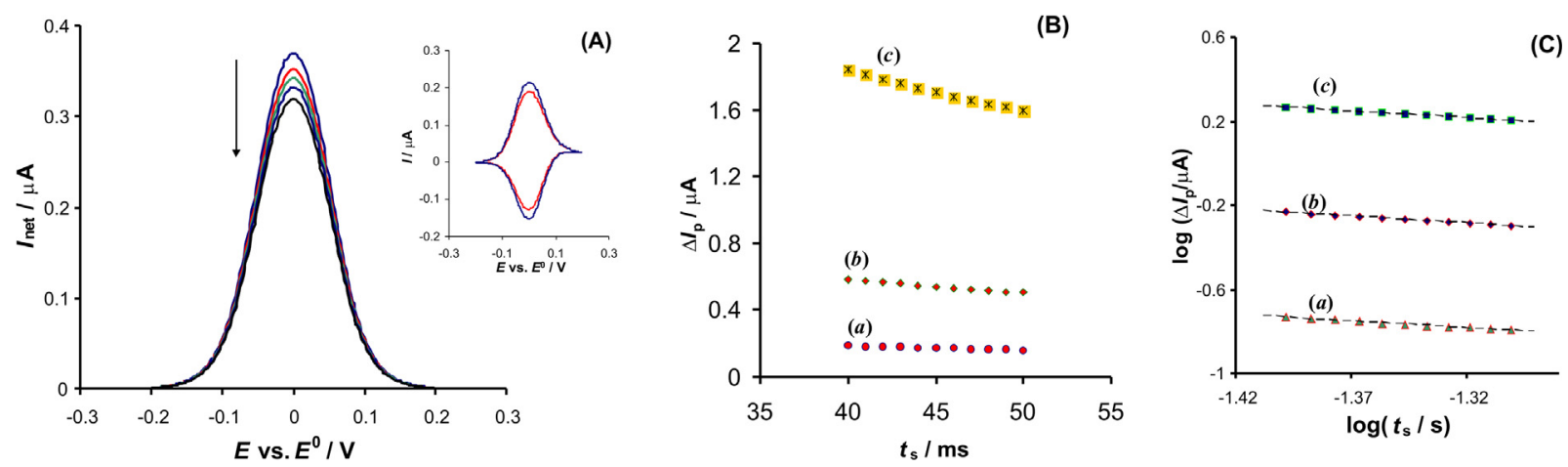

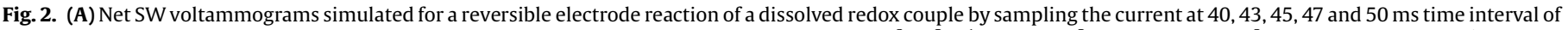

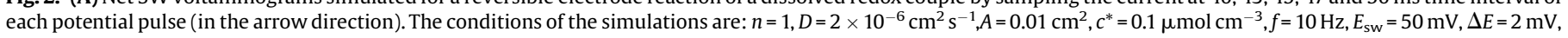

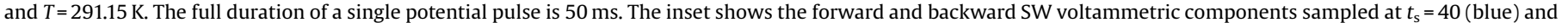

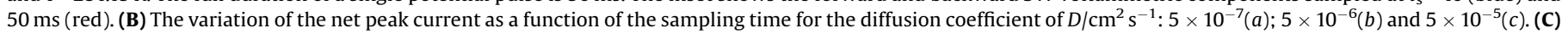
The same data from panel (B) plotted in a log-log relationship. 
intrinsic properties of a given electrode mechanism [5]. In the case of a reversible electrode reaction the $\Psi$ function has the simplest form, being dependent on the dimensionless time and potential only, $\varphi=\left(E-E^{0}\right) n F / R T$ [5], where $E^{0}$ denotes the formal potential of electrode reaction (other symbols have their common meaning). Thus, the presented $\log$ arithmic functions $\log \left(\Delta I_{\mathrm{p}}\right)$ vs. $\log \left(t_{\mathrm{s}}\right)$ in Fig. 2C $\left(\log \left(\Delta I_{\mathrm{p}}\right)=\log \left[n F A c^{*}(D f)^{0.5}\right]+\log \Psi\right)$ indeed reflect the variation of the dimensionless function $\Psi$ with the sampling time, while the influence of the amperometric constant is hidden in the intercept of the line. Moreover, as $\Psi$ depends on $\varphi$, it is plausible to assume that the pulse height would have an effect of the slope of the lines. Simulations conducted at varying amplitudes from 10 to $100 \mathrm{mV}$ revealed that the slope of the function $\log \left(\Delta I_{\mathrm{p}}\right)$ vs. $\log \left(t_{\mathrm{s}}\right)$ varies negligibly from -0.647 (for $E_{\mathrm{sw}}=10 \mathrm{mV}$ ) to -0.6531 (for $E_{\mathrm{sw}}=100 \mathrm{mV}$ ) (data not shown). At this point it might be useful to note that the scan increment $\Delta E$ has a slight influence on the $\log \left(\Delta I_{\mathrm{p}}\right)$ vs. $\log \left(t_{\mathrm{s}}\right)$ function as well, as it affects the total electrolysis time of the experiment through the scan rate of the overall SW modulation $(v=\Delta E f)$. For instance, simulations conducted at $\Delta E=1 \mathrm{mV}$ (the other conditions being identical as for Fig. 2A) are associated with the function slope of -0.6515 , whereas for the scan increment of 10 and $20 \mathrm{mV}$ it is -0.6487 and -0.6392 , respectively.

The foregoing discussion and the data presented in Figs. 2 and 3 do not mean that the proposed methodology is restricted to a potential modulation with a pulse duration of $t_{\mathrm{p}}=50 \mathrm{~ms}(f=10 \mathrm{~Hz})$. The analysis can be expanded by considering the currents from a large portion of the potential pulses and the data can be generalized and presented in a dimensionless form. Specifically, the data can be represented in the form of dimensionless current and dimensionless time fraction, defined as a ratio between the sampling time $t_{s}$ and the pulse duration $t_{p}\left(t_{s} / t_{p}\right)$. The $\log$ - $\log$ relationship between the dimensionless net peak current $\Delta \Psi_{\mathrm{p}}$ of multisampled voltammograms and the dimensionless time fraction over the range from 0.2 to 1 (i.e., considering the last $75 \%$ of the potential pulse) is a line with a slope of -0.673 (simulations for $n=1, E_{\mathrm{sw}}=50 \mathrm{mV}, \Delta E=2 \mathrm{mV}$ ). Thus, the outcome of such analysis is virtually identical as considering the data from the last quarter of the pulse only.

For kinetically controlled electrode reaction of a dissolved redox couple [5], the function $\log \left(\Delta I_{\mathrm{p}}\right)$ vs. $\log \left(t_{\mathrm{s}}\right)$ depends on the electrode kinetics, as illustrated by data in Fig. $3 \mathrm{~A}$, calculated for three different standard rate constant $\left(k_{\mathrm{s}}\right)$ values. This follows from the dependency of the dimensionless function $\Psi$ on the electrode kinetic parameter $\kappa=k_{\mathrm{s}}(D f)^{-0.5}$ [5]. The faster the electrode reaction is the steeper is the decline of the net peak current with the sampling time, due to extensive exhaustion of the diffusion layer with the electroactive material (e.g., curve (a) in Fig. 3A). Detailed, extensive simulations revealed that electrode reactions associated with the rate constant values within the interval $-4 \leq \log \left(k_{\mathrm{s}} / \mathrm{cm} \mathrm{s}^{-1}\right) \leq-1$ could be analysed under conditions of Fig. 3A. In terms of the dimensionless electrode kinetic parameter $\kappa$ the latter interval is defined as $-1.8 \leq \log (\kappa) \leq 1.1$. Thus, the same kinetic interval is accessible either with the present method based on the multisampling procedure of a single voltammogram, or by a classical approach in which a series of conventional voltammograms is required for different frequencies [30]. Besides the net peak current, the kinetic estimation can be performed by analysing the ratio of the forward-to-backward peak currents $\left(I_{\mathrm{p}, \mathrm{f}}\right)$ $\left.I_{\mathrm{p}, \mathrm{b}}\right)$ of $S W$ voltammograms as a function of the sampling time. As shown in Fig. 3B the ratio decreases with sampling time, whereas the slope of the lines increases by increasing the rate of the electrode reaction. The effect is the most pronounced for the reversible electrode reaction (curve $f$ ), being dictated solely by the diffusion of redox species. Let us note that all data presented in Fig. 3 are simulated for the electron transfer coefficient $\alpha=0.5$. Both net peak current and peak current ratio are susceptible to the electron transfer coefficient as well. Thus, for precise estimation of the rate constant previous knowledge of the electron transfer coefficient is required, as common for the most of other kinetic methods. It should be additionally noted that for a slow, electrochemically irreversible electrode reaction, the net peak potential $\left(E_{\mathrm{p}}\right)$ varies slightly with the sampling time. For instance, for $\log \left(k_{\mathrm{s}} / \mathrm{cm} \mathrm{s}^{-1}\right)=-4$, the $E_{\mathrm{p}}$ changes from 0.136 to $0.134 \mathrm{~V}$ vs. $E^{0}$ for the sampling time of 40 to $50 \mathrm{~ms}$, respectively, for simulation conditions corresponding to Fig. 3.

At this point it is useful to note that the foregoing theoretical analysis has been conducted assuming an idealized system free of uncompensated resistance effect [31,32]. According to our previous study in which the uncompensated resistance effect in SWV was studied in detail [32], the resistance for an electrode reaction of solution resident species is affected through the dimensionless parameter $\rho=(n F)^{2} A C^{*} R_{\Omega}(D f)^{1 / 2} / R T$, where $R_{\Omega}$ is the uncompensated resistance. Electrode reaction (1) is affected by the resistance for $\rho>1$. For common experimental conditions when an aqueous supporting electrolyte is used the uncompensated resistance effects is negligible even for pulse duration of $t_{\mathrm{p}}=0.25 \mathrm{~ms}$, i.e. $f=2000 \mathrm{~Hz}$. Such effect should be considered for experiments in non-aqueous medium for $t_{\mathrm{p}}<0.5 \mathrm{~ms}$ (see Table 1 in ref. [32]). Thus, for conditions of Fig. 3, the uncompensated effect should be negligible even for experiments in non-aqueous medium. For instance, simulations conducted in the presence of uncompensated resistance with $\rho=1, k_{\mathrm{s}}=0.01 \mathrm{~cm} \mathrm{~s}^{-1}$, and other conditions being equivalent as for Fig. 3 , revealed that the slope of the line $\log \left(\Delta I_{\mathrm{p}}\right)$
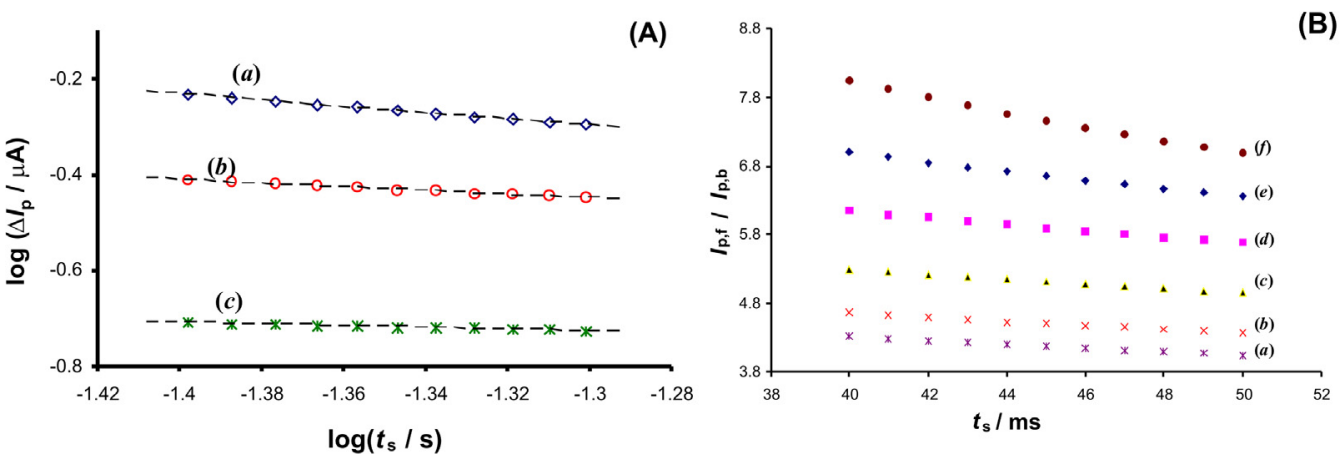

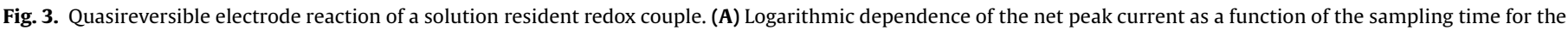

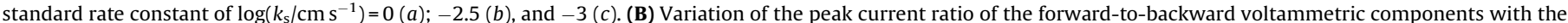

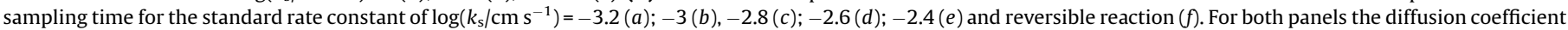
and the electron transfer coefficient are $5 \times 10^{-6} \mathrm{~cm}^{2} \mathrm{~s}^{-1}$ and $\alpha=0.5$, respectively. The other conditions are the same as for Fig. 2 . 
vs. $\log \left(t_{\mathrm{s}}\right)$ is equivalent as in the absence of the uncompensated resistance.

Coupling the electrode reaction with a follow-up chemical reaction results in a remarkable influence to the multisampled SW voltammograms, which is illustrated with the EC' regenerative catalytic mechanism (eqs. (2) and (3)) [23,33,34].

$\mathbf{R}(\mathrm{sol}) \rightleftarrows \mathbf{O}(\mathrm{sol})+\mathrm{e}$

$\mathbf{O}(\mathrm{sol})+\mathbf{S}(\mathrm{sol}) \rightarrow \mathbf{R}(\mathrm{sol})$

The electrode reaction (eq. (2)) is assumed to be electrochemically quasireversible, whereas the coupled chemical reaction (eq. (3)) is chemically irreversible, associated with a pseudo-first order rate constant $k_{\mathrm{c}}\left(\mathrm{s}^{-1}\right)$ [33]. As previously known, the voltammetric properties of the EC' mechanism under conventional SWV are controlled by the electrode kinetic parameter $\kappa$ and the catalytic parameter $\gamma=k_{\mathrm{c}} / f$. In the context of the present study, when single voltammogram is analyzed, both parameters $\kappa$ and $\gamma$ are kept constant. In contrast, in a conventional SWV analysis by varying the SW frequency, both parameters are simultaneously altered, affecting the response in a complex manner. Fig. 4 illustrates the effect of the chemical reaction rate on the evolution of the net peak current of multisampled SW voltammograms with the sampling time. Let us recall that the regenerative chemical reaction (eq. (3)) compensates for the exhaustion of the diffusion layer with the initial electroactive reactant $\mathbf{R}$, causing the slope of the function log $\left(\Delta I_{\mathrm{p}}\right)$ vs. $\log \left(t_{\mathrm{s}}\right)$ to decrease by increasing the rate of the chemical reaction. At a significantly high chemical reaction rate, e.g., log $\left(k_{\mathrm{c}}\right)=1.5$ (curve $e$ in Fig. 4) the net-peak current value for each sampling time $t_{\mathrm{s}} \geq 40 \mathrm{~ms}$ is constant. In addition, the forward and backward components of the response sampled at 40 and $50 \mathrm{~ms}$, for the conditions of curve $e$, are virtually identical (data not shown). Simulations conducted at fixed $k_{\mathrm{c}}$ and varying $k_{\mathrm{s}}$ over the interval $\log \left(k_{\mathrm{s}} / \mathrm{cm} \mathrm{s}^{-1}\right) \leq-4$ (slow electrode reaction) revealed that the effect of the electrode reaction kinetics is negligible on the function $\log \left(\Delta I_{\mathrm{p}}\right)$ vs. $\log \left(t_{\mathrm{s}}\right)$. Thus, the decrease of the net peak current with sampling time is predominantly controlled by the kinetics of the catalytic chemical reaction (eq. (3)), enabling estimation of $k_{\mathrm{c}}$ without previous knowledge of the standard rate

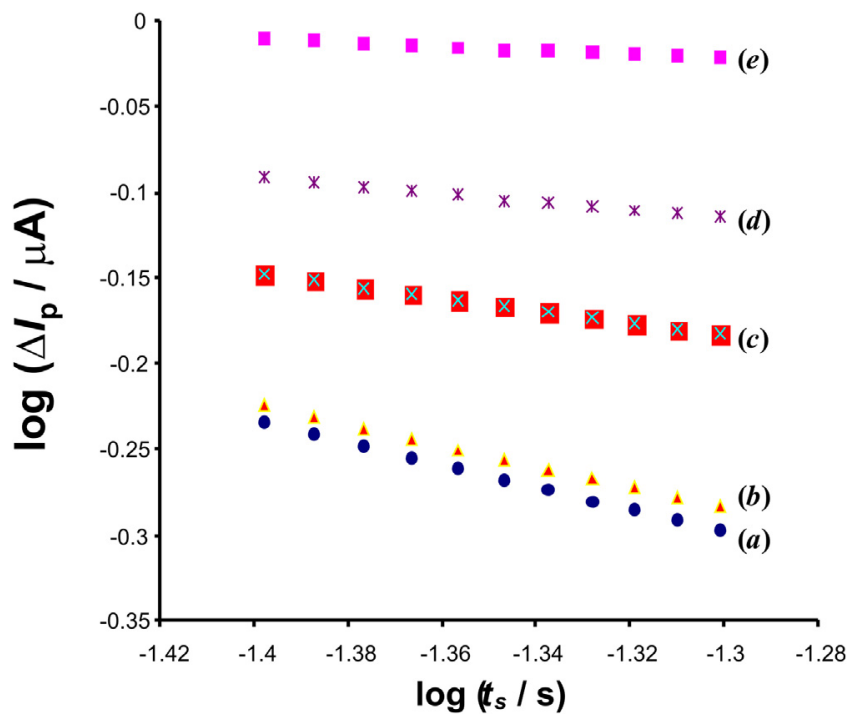

Fig. 4. EC' catalytic mechanism of solution resident species. The log-log dependence of the net peak current on the sampling time, for the catalytic rate constant of log $\left(k_{\mathrm{c}} / \mathrm{s}^{-1}\right)=-5(a) ; 0(b), 1(c) ; 1.25(d)$ and $1.5(e)$. The electron transfer coefficient is $\alpha=0.5$ and the standard rate constant of the electrode reaction is $k_{\mathrm{s}}=10 \mathrm{~cm} \mathrm{~s}^{-1}$. The other conditions are the same as for Fig. 2. constant $k_{\mathrm{s}}$. However, over the interval $-4<\log \left(k_{\mathrm{s}} / \mathrm{cm} \mathrm{s}^{-1}\right)<1$ (quasireversible electrode reaction) the slope of the function increases by increasing the rate of the electrode reaction. Thus, for a quasireversible electrode reaction, besides diffusion, surface concentrations of the redox species depend on both electrode and chemical reaction.

As can be expected from the previous knowledge of the specific chronoamperometric properties of surface confined ((sur) in eq. (4)) electrode processes [5], multisampling procedure results in a dramatic evolution of corresponding voltammograms with the sampling time.

$\mathbf{R}($ sur $) \rightleftarrows \mathbf{O}($ sur $)+\mathrm{e}$

Fig. $5 \mathrm{~A}$ depicts the variation of the net peak currents with the sampling time for three values of the surface rate constant $\left(k_{\mathrm{s}, \mathrm{sur}}\right)$ of the electrode reaction, considering almost the full duration of the potential pulses. As the response is not affected by the diffusional mass transport, the net peak current decreases drastically with the sampling time. Consequently, the fastest electrode reaction results in the lowest net peak current sampled at the end of the potential pulse, which corresponds to the conventional SW voltammogram. As obvious from Fig. 5A, the gradient of the net peak current decrease with the sampling time is markedly different, being a function of the standard rate constant, which is in agreement with the previous findings [5]. Interestingly, analysing the product of the net peak current and the corresponding sampling time $\left(\Delta I_{\mathrm{p}} t_{\mathrm{s}}\right)$ vs. the sampling time a parabolic function is obtained, as depicted in the panel 5B. The product $\Delta I_{\mathrm{p}} t_{\mathrm{s}}$ has a unit of the electrical charge, though it is not directly correlated with the charge consumed in the course of the complex SW voltammetric experiment. The parabolic functions from the panel $5 \mathrm{~B}$ represents a sort of quasireversible maximum, the wellknown phenomenon in conventional SWV of surface confined electrode processes $[2,5,30]$. The positions of presented quasireversible maxima are related with an optimal sampling time that provides synchronisation between the sampling time and the rate of the electrode reaction. The quasireversible maxima are associated with a critical sampling time $\left(t_{\mathrm{s}, \mathrm{crit}}\right)$ the value of which can be easily used for estimation of the standard rate constant, as follows from the linear relationship between $k_{\mathrm{s}, \text { sur }}$ and $t_{\mathrm{s}, \mathrm{crit}}$ presented in the inset of panel $5 \mathrm{~B}$.

Besides the quasireversible maximum, the kinetic analysis of the surface confined electrode reaction can be carried out by measuring the peak potential separation $\left(\Delta E_{\mathrm{p}}\right)$ of the forward-tobackward components of the response as a function of the sampling time, as shown in Fig. 5C. In agreement with previous methods, the peak potential separation is a linear function of log $\left(t_{\mathrm{s}}\right)$, the slope and the intercept of the function being dependent on the rate constant (Fig. 5C).

Finally, the phenomenon of splitting of the net SW peak can be observed only by changing the sampling time for a constant set of values of the applied SW frequency and amplitude, as illustrated by Fig. $6[3,35,36]$. Net peak splitting, which is a unique feature of a surface confined redox system, emerges by increasing the sampling time, being in agreement with previous findings $[2,29]$. For a given set of the amplitude and the frequency, a critical sampling time can be defined at which the splitting commences, which is a function of the rate constant of the studied electrode reaction. For instance, for the conditions of Fig. 6 the splitting emerges at $t_{\mathrm{s}}=38 \mathrm{~ms}$. If the rate constant is lower, i.e. $k_{\mathrm{s}}$, sur $=30$ and $25 \mathrm{~s}^{-1}$, the splitting is observed at sampling time of $t_{\mathrm{s}}=42$ and $48 \mathrm{~ms}$, respectively. Thus, an analogous methodology to the previously reported one $[2,3,21,35,36]$ can be developed for kinetic and mechanistic characterization of the electrode reaction by recording a single experiment only. 
(A)

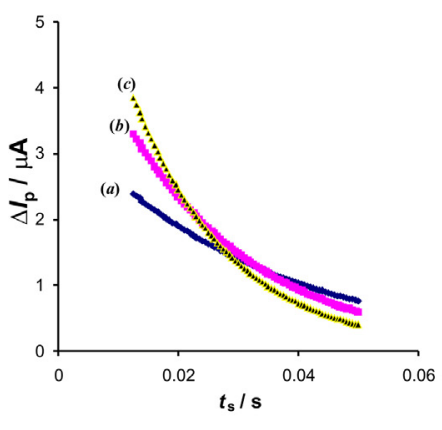

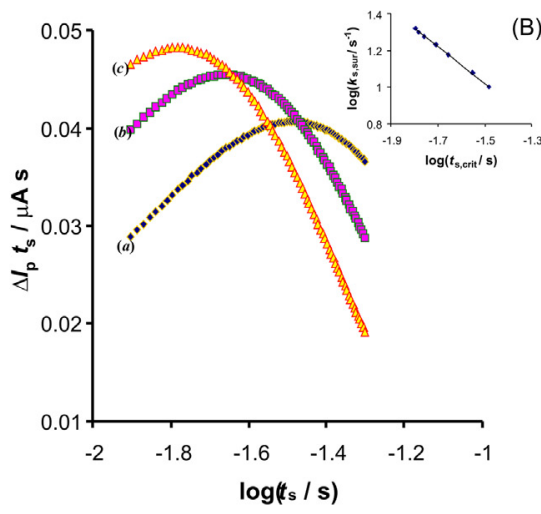

(B)

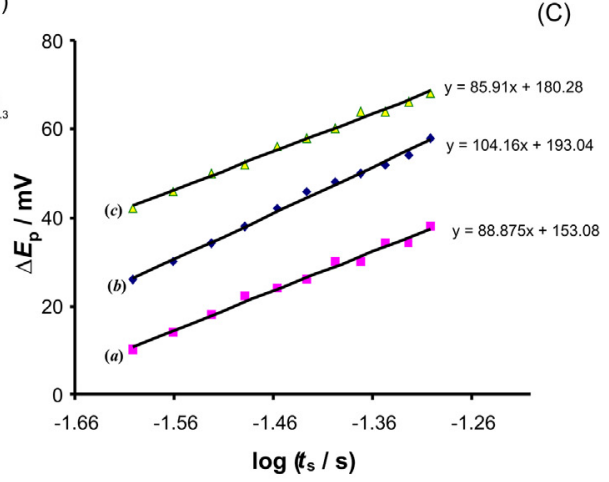

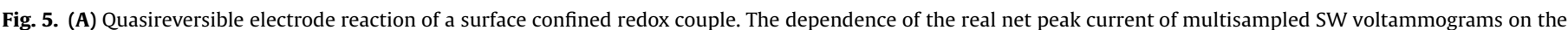

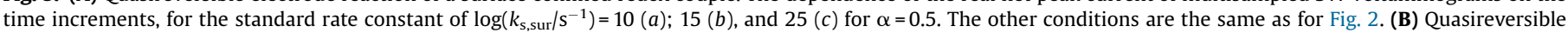

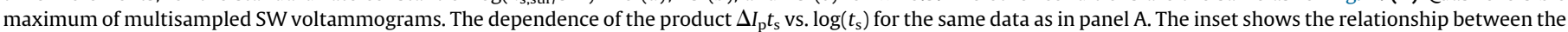

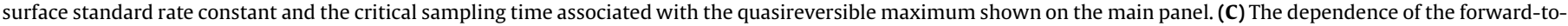
backward peak potential separation as a function of the time increments for the standard rate constant of $\log \left(k_{\mathrm{s}, \mathrm{sur}} / \mathrm{s}^{-1}\right)=10(a) ; 20(b)$, and $30(c)$.
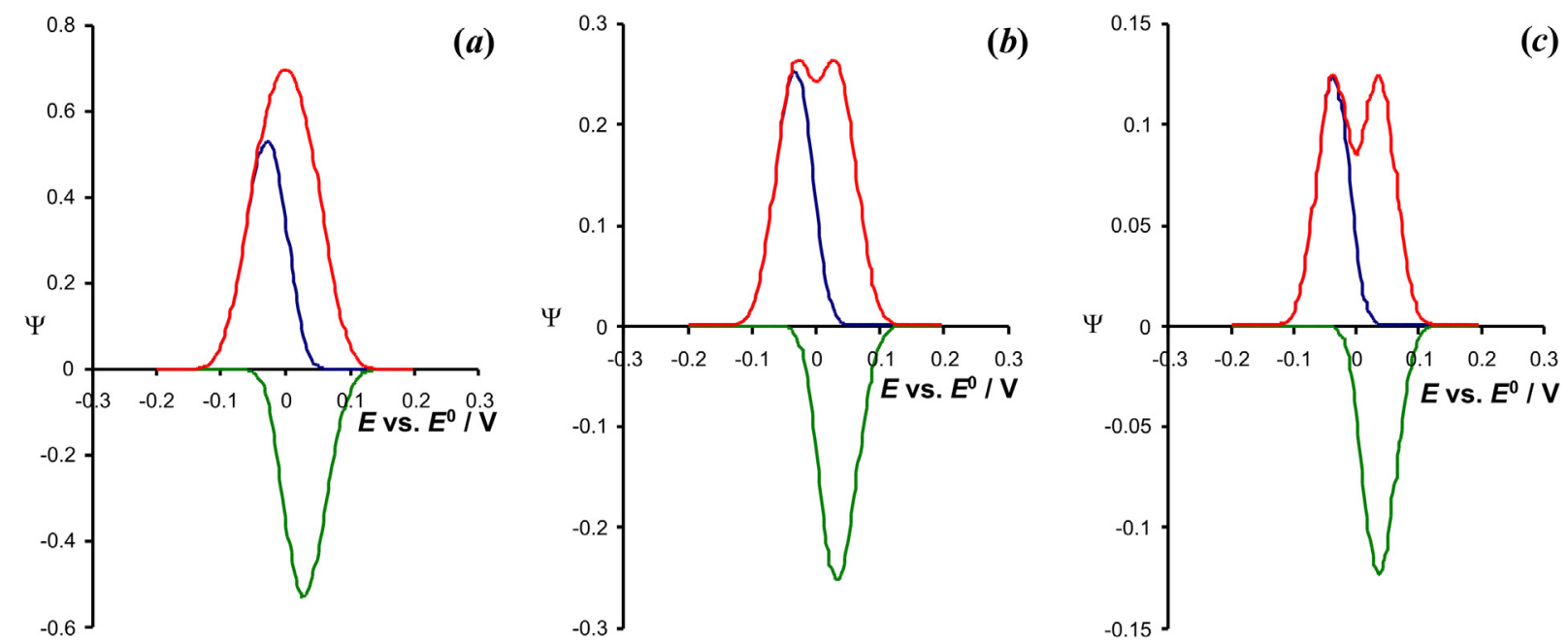

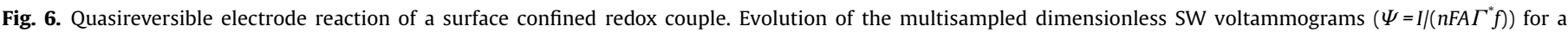

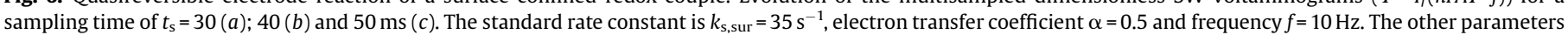
of the potential modulation are the same as for Fig. 2 .

\subsection{Experimental data}

The foregoing methodology can be readily applied in the analysis of electrode reactions. The voltammetric responses of the trivalent europium cation [27] and azobenzene [29] serve as examples for a quasireversible electrode reaction of a dissolved and surface confined redox couple, respectively.

Typical conventional SW voltammetric response of $\mathrm{Eu}^{3+}(\mathrm{aq})$ reduction at HMDE is depicted in Fig. 7A. The features of the response referring to the net half-peak width $\left(\Delta E_{\mathrm{p} / 2}=207 \mathrm{mV}\right)$, the forward to backward peak current ratio $\left(\left|I_{\mathrm{p}, \mathrm{f}}\right| I_{\mathrm{p}, \mathrm{b}} \mid=2.74\right)$ and the peak potential separation $\left(\Delta E_{\mathrm{p}}=71 \mathrm{mV}\right)$ are typical for a quasireversible electrode reaction [5]. The frequency analysis over the interval from 8 to $160 \mathrm{~Hz}$ shows a nonlinear dependence of $\Delta I_{\mathrm{p}}$ vs. $f^{0.5}$ (data not shown) and a linear dependence of the net peak potential $E_{\mathrm{p}}$ vs. $\log (f)$, being in agreement with the theoretical predictions for a kinetically controlled electrode reaction [5]. A corresponding scan rate analysis under conditions of cyclic voltammetry (CV) over the interval from $v=20$ to $1000 \mathrm{mV} / \mathrm{s}$ reveals a linear dependence of both cathodic and anodic peak currents on the square root of the scan rate (linear regression coefficint is $R^{2}=0.984$ and $R^{2}=0.986$, respectively). The $\log -\log$ relationship of the peak current and the scan rate is associated with a linear regression function and a slope of 0.48 and 0.56 for the cathodic and anodic peak currents, respectively, implying that mass transfer is of diffusional nature, being unaffected by adsorption phenomena of either reactant or product of the electrode reaction $[37,38]$. At the same time, peak potentials vary with the scan rate, and the peak potential separation increases from $146 \mathrm{mV}$ to $267 \mathrm{mV}$ for $v=40$ and $1000 \mathrm{mV} / \mathrm{s}$, respectively. The peak current increases linearly with increasing europium ion concentration from 0.1 to $2 \mathrm{mmol} \mathrm{L}^{-1}$ both in CV and SWV. At the same time the peak potential remains almost constant with negligible shift toward more negative values ( $9 \mathrm{mV}$ in $\mathrm{SWV}$ ).

Following the foregoing basic voltammetric characterization of the electrode reaction, a detailed kinetic analysis has been performed by varying the SW amplitude (over the interval from 20 to $250 \mathrm{mV}$ ) and measuring the peak potential separation between the forward to backward SW voltammetric components 
$[3,35,36]$ and amplitude-based quasireversible maximum [2]. A careful inspection of the amplitude normalized net peak currents reveals the presence of an amplitude-based quasireversible maxima for the measurements conducted at 8, 25, 50 and $100 \mathrm{~Hz}$ (data not shown) implying that electrode reaction is kinetically controlled at all used frequencies [2]. The overall kinetic analysis gives a standard rate constant value of $k_{\mathrm{s}}=(0.52 \pm 0.04)$ $\times 10^{-3} \mathrm{~cm} \mathrm{~s}^{-1}$. The latter is an average of the kinetic estimations based on the amplitude-based quasireversible maximum [2] and the component peak potential separation method of conventional and potential-corrected SW voltammograms [3,35,36].

As noted in the experimental section, the multisampling procedure in SWV is not directly accessible with most of the present potentiostat used in a SWV mode. This drawback was circumvented by performing a series of repetitive double-step chronoamperometric experiments at a single mercury drop in a way to reproduce a SWV potential modulation. The experiments have been performed with a pulse of $50 \mathrm{mV}$ height and duration of $t_{\mathrm{p}}=62.5 \mathrm{~ms}$, corresponding to a SWV modulation with the frequency of $f=8 \mathrm{~Hz}$, amplitude $E_{\mathrm{sw}}=50 \mathrm{mV}$ and scan increment $\Delta E=5 \mathrm{mV}$. The currents from the last portion of each potential pulse have been analysed, i.e. the sampling time was $t_{\mathrm{s}} \geq 50 \mathrm{~ms}$, and a series of corresponding multisampled SW voltammograms have been constructed (see Fig. 7B) out of a single experiment. As predicted by the theory multisampled net SW voltammograms decreases by increasing the sampling time, while the net peak potential and half peak width remain virtually constant (Fig. 7B). The $\log$-log dependence of the net peak currents of multisampled $\mathrm{SW}$ voltammograms vs the sampling time is a line with a slope of -0.45 . Note that for a reversible electrode reaction the theory predicts a slope of -0.65 , whereas for a quasireversible reaction the slope is lower in absolute values, which indicates a quasireversible nature of the studied electrode reaction of $\mathrm{Eu}^{3+}$ reduction. The estimation of the rate constant was performed by fitting the experimental with the theoretical data of multisampled SW voltammograms as a function of the sampling time (Fig. 7C). For consistent comparison an additional normalization of both experimental and theoretical data has been done with respect to the net peak current of the voltammogram measured at $t_{\mathrm{s}}=50 \mathrm{~ms}$. The best fit was obtained for a rate constant of $k_{\mathrm{s}}=1.7 \times 10^{-3} \mathrm{~cm}$ $\mathrm{s}^{-1}$, which is in accord to the previous value obtained by the amplitude based analysis of conventional SW voltammograms. In the fitting procedure, the selected values for the electron transfer coefficient $(\alpha=0.65)$ and the diffusion coefficient $\left(D=1 \times 10^{-6}\right.$ $\mathrm{cm}^{2} \mathrm{~s}^{-1}$ ) are close to those reported in the literature for the electrode reaction of $\mathrm{Eu}^{3+}[26-28]$.
An analogous analysis has been conducted with multisampled SW voltammograms for the azobenzene electrode reaction as an example of a surface confined redox system [39]. The general electrochemical behaviour of this system is already well-known and frequently used for illustration of theoretical models referring to simple surface confined electrode reaction [29]. Similar to the previous experimental system, the kinetic characterisation of the electrode reaction in a phosphate buffer at $\mathrm{pH}=4.8$ was performed by applying conventional SWV according to the previous amplitude based methods [2,3]. Note that the amplitude-based quasireversible maximum was not observed and only the descending part of the dependence $\Delta I_{\mathrm{p}} / E_{\mathrm{sw}}$ vs. $\log \left(E_{\mathrm{sw}}\right)$ was found for the measurements at 8,25 and $50 \mathrm{~Hz}$, indicating relatively slow electrode kinetics. Analysis of the voltammetric components peak potential separation as a function of the amplitude led to the estimation of the standard rate constant with an average value of $k_{\text {sur }}=2.4 \pm 0.4 \mathrm{~s}^{-1}$.

The evolution of the multisampled SW voltammograms of azobenzene are in full qualitative agreement with the theoretical predictions. Increasing the sampling time from 50 to $62.5 \mathrm{~ms}$ results in decreasing of the net peak current of corresponding voltammograms from 1.454 to $1.151 \mu \mathrm{A}$, respectively for experiments with $2 \mu \mathrm{mol} \mathrm{L}^{-1}$ azobenzene and accumulation time of $60 \mathrm{~s}$. Normalization of the multisampled SW voltammograms with the corresponding sampling time leads to an appearance of a sort of quasireversible maximum, as predicted by the theory (cf. Fig. 5B). Fitting the position of the experimental with the theoretical quasireversible maximum is found for the standard rate constant $k_{\mathrm{s}, \mathrm{sur}}=2.34 \mathrm{~s}^{-1}$ and $\alpha=0.5$ (Fig. 8A), which is in excellent agreement with the previously estimated value. Besides the net peak current, the peak potential separation of the experimental multisampled SW voltammograms of azobenzene is a function of the sampling time as well. The best fit with the theoretical data is found for $k_{\mathrm{s}, \mathrm{sur}}=2.85 \mathrm{~s}^{-1}$ (Fig. 8B) which also agrees well with the previous values, confirming the applicability of the proposed methodology.

\section{Conclusion}

It has been demonstrated that an advanced time-domain analysis of a single repetitive double-step chronoamperometric experiment, with a potential protocol being equivalent to SWV, provides a new means for characterization of an electrode reaction. Conducting the proposed methodology with a potential modulation with relatively low frequency (i.e. $f \leq 200 \mathrm{~Hz}$; potential pulse duration $\left.t_{\mathrm{p}} \geq 2.5 \mathrm{~ms}\right)$ and a low scan increment $(\Delta E \leq 10 \mathrm{mV})$,
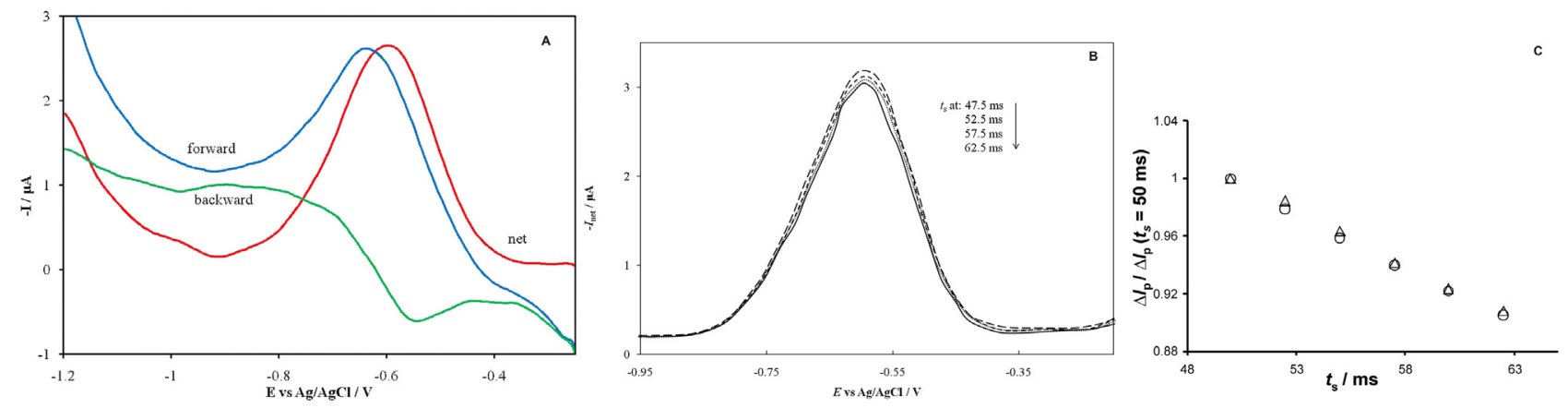

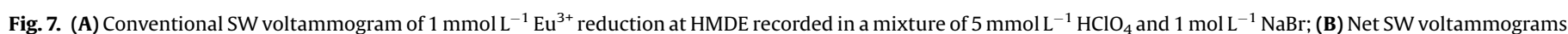

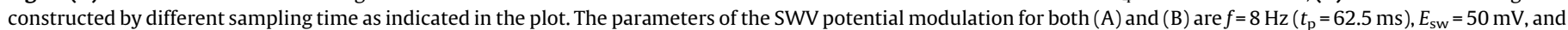

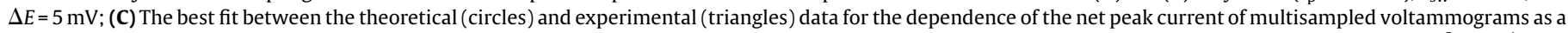

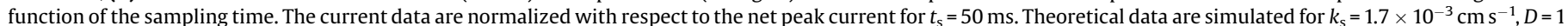
$\times 10^{-6} \mathrm{~cm}^{2} \mathrm{~s}^{-1}$, and $\alpha=0.65$. 

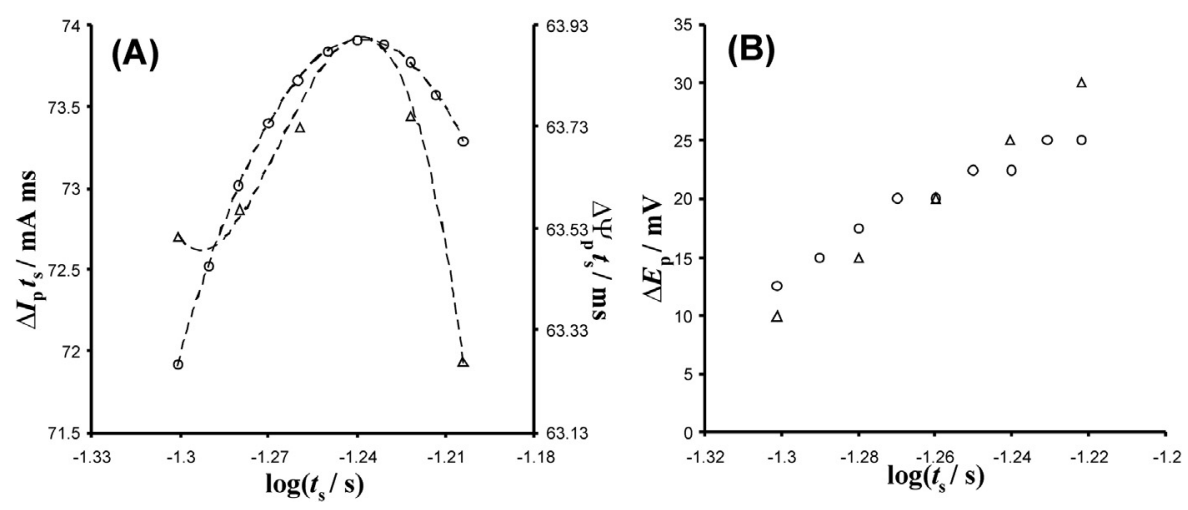

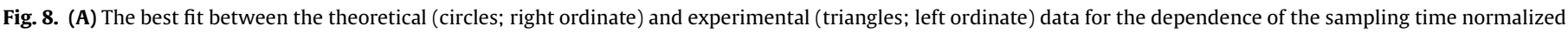

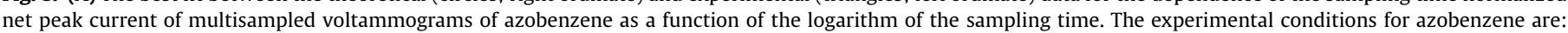

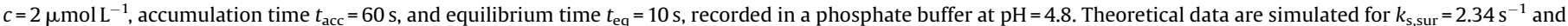

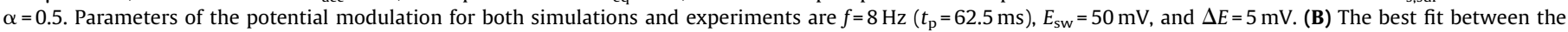

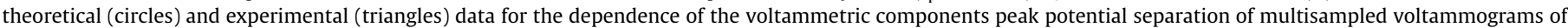
azobenzene as a function of the logarithm of the sampling time. The best fit found for $k_{\mathrm{s}, \mathrm{sur}}=2.85 \mathrm{~s}^{-1}$. All other conditions are identical as for panel (A).

ensures the charging current to be negligible in the last quarter period of the potential pulse, when the multisampling current procedure is proposed. Besides, for common experimental conditions [32], the latter experiment is expected to be virtually unaffected by the uncompensated resistance due to the low scan rate, thus providing a high quality electrochemical data of the faradaic electrode process only. Yet, the proposed methodology for kinetic characterisation can be conducted with much shorter potential pulses, and is not necessary limited to the last quarter of potential pulses, in spite of the expected contribution of the charging current [5].

When single experiment is conducted at a given frequency, all frequency related kinetic parameters in the current function $\Psi$ typical for the studied electrode mechanism are fixed, which makes the analysis simple and the estimated kinetic parameters by the fitting procedure reliable. The latter is not the case in conventional analysis in which the frequency is commonly varied, causing simultaneous variation of all frequency related parameters, which results in a complex variation of the current function $\Psi$. Currently, we propose estimation of the kinetic data by fitting the experimental with simulated data for a given electrode mechanism. In the subsequent work, we aim to propose simplified semi-empirical formulas for kinetic estimation.

It should be finally noted that in the real experimental analysis, prior application of the proposed methodology, one cannot avoid preliminary general characterisation of the electrode mechanism, in which recording of a series of voltammograms for different experimental conditions and instrumental parameters is indispensable, e.g., selecting the most appropriate potential time duration, determination of the order of the electrode reaction, determination the possible uncompensated resistance effect etc. Moreover, rigorously speaking, the application of the proposed methodology for kinetic characterization of the electrode reaction, a previous knowledge of the type of the electrode mechanism is required. Yet, the proposed method is conceptually new, the implementation of which in the modern digital electrochemical instrumentation is expected to be simple, resulting in a new form of a SWV analysis for advanced and fast characterization of electrode processes.

\section{Acknowledgements}

VM acknowledges with the gratitude the financial support of the DAAD foundation through the multilateral project
"International Masters and Postgraduate Programme in Materials Science and Catalysis" (MatCatNet) and the COST project CM1302 SIPs. DG acknowledges the support from the National Science Centre of Poland through the grant 2011/03/N/ST4/01338. IB acknowledges the support of "Deutsche Forschungsgemeinschaft" though the SFB 1027 Project C4 and the individual research grant B03643/3-1.

\section{References}

[1] F. Scholz, Electroanalytical Methods: Guide to Experiments and Applications, Springer, Berlin Heidelberg, 2009.

[2] V. Mirčeski, E. Laborda, D. Guziejewski, R.G. Compton, Anal. Chem. 85 (2013) 5586-5594.

[3] V. Mirčeski, D. Guziejewski, K. Lisichkov, Electrochim. Acta 114 (2013) 667673.

[4] D.K. Gosser, Cyclic voltammetry: simulation and analysis of reaction mechanisms, VCH, 1993.

[5] V. Mirčeski, S. Komorsky-Lovric, M. Lovric, Square-Wave Voltammetry Theory and Application, Springer-Verlag, Berlin Heidelberg, 2007.

[6] V. Mirčeski, R. Gulaboski, Maced. J. Chem. Chem. Eng. 33 (2014) 1-12.

[7] V. Mirčeski, R. Gulaboski, M. Lovric, I. Bogeski, R. Kappl, M. Hoth, Electroanalysis 25 (2013) 2411-2422.

[8] A. Molina, J. González, E. Laborda, R.G. Compton, J. Electroanal. Chem. 756 (2015) 1-21.

[9] C. Batchelor-Mcauley, E. Kätelhön, E.O. Barnes, R.G. Compton, E. Laborda, A. Molina, ChemistryOpen 4 (2015) 224-260.

[10] M.A. Mann, J.C. Helfrick, L.A. Bottomley, Anal. Chem. 86 (2014) 8183-8191.

[11] M.A. Mann, L.A. Bottomley, Langmuir 31 (2015) 9511-9520.

[12] L. Čižmek, Š. Komorsky-Lovrić, M. Lovrić, ChemElectroChem 2 (2015) 20272031.

[13] M. Lovrić, Š. Komorsky-Lovrić, J. Electroanal. Chem. 735 (2014) 90-94.

[14] D. Jadreško, M. Zelić, Electroanalysis 27 (2015) 1669-1675.

[15] E. Zabost, W. Liwinska, M. Karbarz, E. Kurek, M. Lyp, M. Donten, Z. Stojek, Bioelectrochem. 109 (2016) 1-8.

[16] R.G. Compton, C.E. Banks, Understanding Voltammetry, 2nd edition, Imperial Coll Press, Covent Garden, 2011, pp. 1-429.

[17] E. Laborda, J. González, Á. Molina, Electrochem. Commun. 43 (2014) 25-30.

[18] V. Mirčeski, D. Guziejewski, W. Ciesielski, Electroanalysis 23 (2011) 1365-1375.

[19] S. Skrzypek, V. Mirčeski, S. Smarzewska, D. Guziejewski, W. Ciesielski, Coll. Czech. Chem. Commun. 76 (2011) 1699-1715.

[20] V. Mirčeski, D. Guziejewski, R. Gulaboski, Maced. J. Chem. Chem. Eng. 34 (2015) $181-188$.

[21] A.M. Bond, D. Elton, S.-X. Guo, G.F. Kennedy, E. Mashkina, A.N. Simonov, J. Zhang, Electrochem. Commun. 57 (2015) 78-83.

[22] L. Ramaley, M.S. Krause, Anal. Chem. 41 (1969) 1365-1369.

[23] V. Mirčeski, R. Gulaboski, Electroanalysis 13 (2001) 1326-1334.

[24] R. Gulaboski, V. Mirčeski, Electrochim. Acta 167 (2015) 219-225.

[25] F. Quentel, V. Mirčeski, A. Laouenan, C. Elleouet, C.L. Madec, Electroanalysis 15 (2003) 270-277.

[26] Y. Wang, E. Laborda, M.C. Henstridge, F. Martinez-Ortiz, A. Molina, R.G. Compton, J. Electroanal. Chem. 668 (2012) 7-12.

[27] M. Zelić, Croat. Chem. Acta 76 (2003) 241-248.

[28] A. Ribeiro, A. Valente, V. Lobo, Chem. Chem. Technol. 5 (2011) 133-138. 
[29] V. Mirčeski, M. Lovrić, Electroanalysis 9 (1997) 1283-1287.

[30] V. Mirčeski, M. Lovrić, Electroanalysis 11 (1999) 984-989.

[31] D. Krulic, N. Fatouros, J. Electroanal. Chem. 652 (2011) 26-31.

[32] V. Mirčeski, M. Lovric, J. Electroanal. Chem. 497 (2001) 114-124.

[33] V. Mirčeski, A. Bobrowski, J. Zarebski, F. Spasovski, Electrochim. Acta 55 (2010) 8696-8703.

[34] V. Mirčeski, R. Gulaboski, J. Solid State Electrochem. 7 (2003) 157-165.
[35] V. Mirčeski, S. Smarzewska, D. Guziejewski, Electroanalysis 28 (2016) 385393.

[36] D. Guziejewski, V. Mirčeski, D. Jadresko, Electroanalysis 27 (2015) 67-73.

[37] D. Guziejewski, M. Brycht, A. Nosal-Wiercińska, S. Smarzewska, W. Ciesielski, S. Skrzypek, J. Environ. Sci. Health B 49 (2014) 550-556.

[38] D. Altunöz-Erdoğan, N. Erk, E. Kılıç, Cent. Eur. J. Chem. 11 (2013) 706-716.

[39] F. Quentel, V. Mirčeski, Electroanalysis 15 (2003) 1787-1794. 